\title{
Hydro2k: Integrating proxy data and models for insights into past and future hydroclimate
}

\author{
Palisades, USA, 1-3 June 2016
}

Jason E. Smerdon', J. Luterbacher ${ }^{2}$ and S.J. Phipps ${ }^{3}$

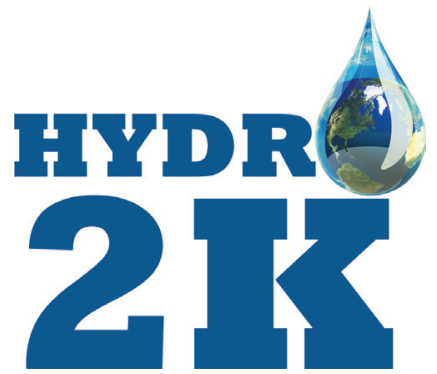

\begin{abstract}
New paleoclimate reconstructions are expanding insights into hydroclimate variability and change during the Common Era (e.g. Cook et al. 2015; Diaz and Wahl 2015). Many last-millennium simulations using fully-coupled climate models are also becoming available (e.g. Fernandez-Donado et al. 2013). Importantly, Phase 3 of the Paleoclimate Modelling Intercomparison Project (PMIP3) included a last-millennium experiment in its protocol, and multiple centers performed the experiment using the same state-of-the-art models used for Phase 5 of the Coupled Model Intercomparison Project (CMIP5). Ensembles of last-millennium simulations (e.g. Otto-Bliesner et al. 2016) are also proposed as part of PMIP4. These developments make the time ripe for expanding our understanding of past hydroclimate variability, while exploring whether climate models simulate hydroclimate in ways that are consistent with the paleoclimate record.
\end{abstract}

Addressing these emerging themes was the focus of a workshop titled Comparing data and model estimates of hydroclimate variability and change over the Common Era, organized jointly by PAGES $2 \mathrm{k}$ and the PAST2k working group of PMIP (see Schmidt et al. 2014 and PAGES 2k-PMIP3 group 2015 for outcomes of related past workshops). The workshop included presentations from paleoclimatologists on hydroclimate proxies and their interpretation, climate modelers on current efforts to simulate climate over the Common Era, and from researchers working at the interface of these two areas (see the full agenda and presentation slides at: http:// pages2kpmip3.github.io/). The presentations and associated discussions provided an assessment of the state of the science, the challenges associated with hydroclimate comparisons between proxy information and model simulations, and an evaluation of best practices in this emerging field.

Workshop discussions underscored the nascent stage of data-model comparisons over the Common Era generally, and specifically with regard to hydroclimate. Recommendations include the need to account for internal variability and its influence on comparison outcomes. Internal variability will obscure comparisons between proxy reconstructions and climate model simulations, even if they represent both forced changes and internal variability perfectly (Fig. 1). Analyses that go beyond simple time-series associations are therefore

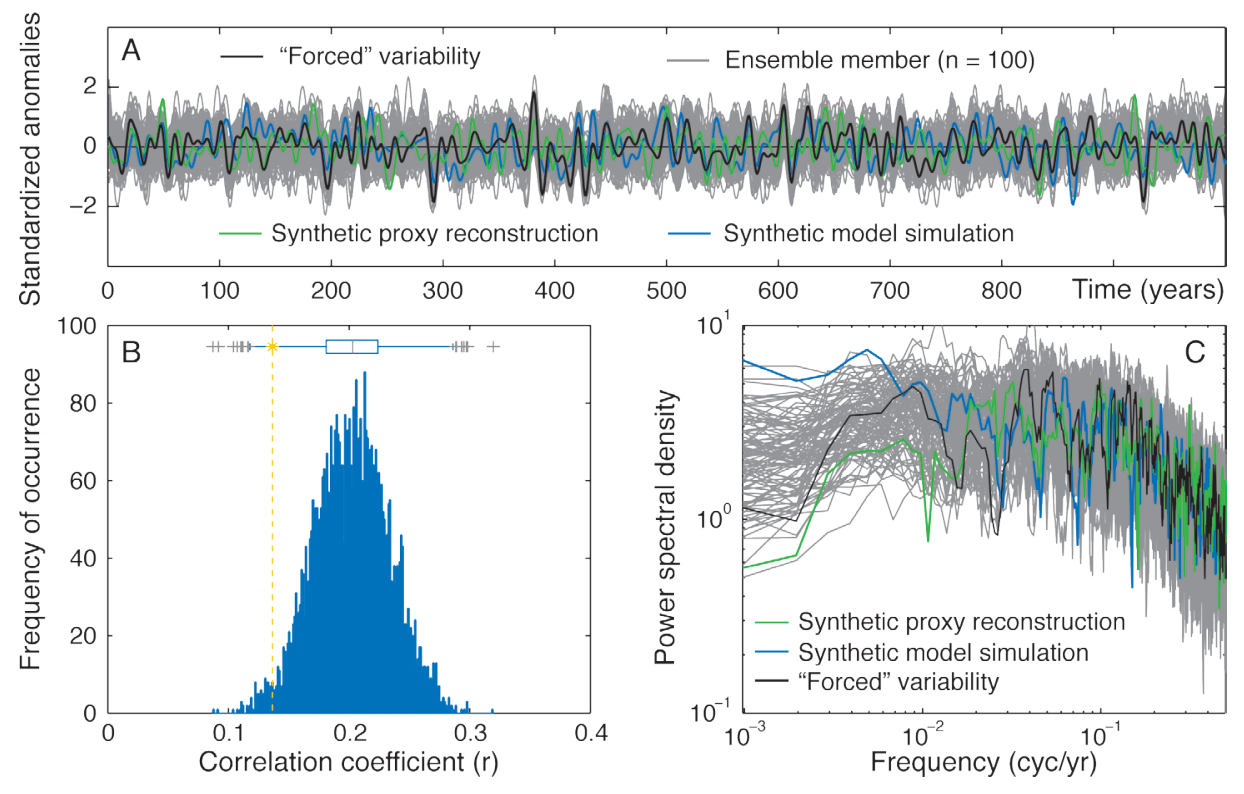

Figure 1: (A) A "forced" signal modeled as an autoregressive lag-1 process $(A R(1) ; \mu=0, \sigma=1, \rho=0.3)$ and 100 synthetic climate histories constructed by adding the forced series to $100 \operatorname{AR}(1)$ series $(\mu=0, \sigma=2, \rho=0.3)$ to represent internal variability. Two of the ensemble members are highlighted to represent a proxy record and a model simulation that both perfectly sample the forced climate variability while having different realizations of internal variability. All plotted series have been processed with a 10-year lowpass filter. (B) The distribution of Pearson's correlation coefficients calculated between each of the grey series in panel (A). The boxplot shows the median, interquartile range and the outliers in grey crosses. The yellow dashed line and star indicate the correlation coefficient calculated between the unfiltered proxy and model series in panel (A). (C) The multitaper power spectral density estimates for the forced series and all grey series shown in panel (A).

strongly advocated, including bootstrapping exercises to evaluate null hypotheses and assessments based on spectral information. The importance of proxy system models (PSMs) was also stressed. When studying decadal and centennial variability, PSMs allow better accounting of the reddening of climatic signals present in some proxy records. For the emerging area of paleoclimate data-assimilation, PSMs also account for the multiple environmental variables often integrated by proxies. Generally, comparison efforts should include as many models as possible, while incorporating the collection of ensemble simulations from individual models. Spatial sampling should be done carefully, keeping in mind that local associations do not necessarily scale regionally or globally (and vice versa). These general points of guidance and the emerging efforts to refine techniques for hydroclimate data-model comparisons will further enhance our ability to evaluate climate models, understand the dynamics of hydroclimate variability and change, and constrain our characterizations of climate risks in the future.

\section{ACKNOWLEDGEMENTS}

This workshop was made possible by PAGES and the Climate Center at the Lamont-Doherty Earth Observatory.

\section{AFFILIATIONS}

'Lamont-Doherty Earth Observatory of Columbia University, Palisades, USA

${ }^{2}$ Department of Geography and Centre for International Development and Environmental Research, Justus-Liebig-University Giessen, Germany ${ }^{3}$ Institute for Marine and Antarctic Studies, University of Tasmania, Hobart, Australia

\section{CONTACT}

Jason Smerdon: jsmerdon@ldeo.columbia.edu REFERENCES

PAGES 2k-PMIP3 group (2015) Clim Past 11: 1673-1699 Cook ER et al. (2015) Sci Adv 1, doi:10.1126/ sciadv.1500561

Diaz HF, Wahl ER (2015) J Clim 28: 4637-4652 Fernandez-Donado L et al. (2013) Clim Past 9: 393-421 Otto-Bliesner BL et al. (2016) BAMS 97: 735-754 Schmidt GA et al. (2014) Clim Past 10: 221-250 